\title{
DocumentaÇ̃̃o
}





\section{O sapo dourado do arquivo pessoal de Mário de Andrade analisado com o método não destrutivo de fluorescência de raios $X$}

Lúcia E. Thome ${ }^{1}$, Márcia A. Rizzutto², Elisabeth M. Kajiya ${ }^{3}$, Pedro

H. O. V. Campos ${ }^{4}$, Carlos R. Appoloni ${ }^{5}$, Fabio Lopes ${ }^{6}$

\section{Introdução}

No arquivo pessoal de Mário de Andrade de salvaguarda do Instituto de Estudos Brasileiros da Universidade de São Paulo (IEB-USP), a coleção de discos fornece documentário de grande interesse e complementa a documentação sonora das coleções dos arquivos do IEB. Os documentos desta coleção são, todos eles, de acetato, alguns possuindo capas originais e, conforme destaca seu proprietário, a metade dos discos foi por ele recebida como presente. Dentre os mais de cem títulos de álbuns musicais destacamos "O sapo dourado" que, além de ser um disco infantil, está classificado como Música Brasileira Erudita. Esse documento sonoro é composto de dois discos cujo conjunto são de artistas líricos (elencados no encarte), coro misto e orquestra não mencionados sob a direção do compositor Hekel Tavares e argumento de Martha Dutra. A locução é de Sérgio A. Vasconcellos e os versos foram produzidos por

I Supervisora técnica do Serviço de Conservação e Restauro, Instituto de Estudos Brasileiros da Universidade de São Paulo (USP, São Paulo, SP, Brasil). E-mail: restauroieb@yahoo.com.br

2 Professora doutora do Departamento de Física Nuclear, Instituto de Física da Universidade de São Paulo (USP, São Paulo, SP, Brasil). E-mail: rizzutto@if.usp.br

3 Técnica do Projeto $\mathrm{CNPq}$ do Departamento de Física Nuclear, Instituto de Física da Universidade de São Paulo (USP, São Paulo, SP, Brasil). E-mail: elizabethkajiya@gmail.com

4 Doutorando do Departamento de Física Nuclear, Instituto de Física da Universidade de São Paulo (USP, São Paulo, SP, Brasil). E-mail: scout_pc@yahoo.com.br

5 Professor associado do Departamento de Física da Universidade Estadual de Londrina (UEL, Londrina, PR, Brasil), Grupo de Física Nuclear Aplicada. E-mail: appoloni@uel.br

6 Doutorando do Grupo de Física Nuclear Aplicada, Departamento de Física da Universidade Estadual de Londrina (UEL, Londrina, PR, Brasil). E-mail: fabiolopes@uel.br 
W. Henrique. Dentre as principais características que diferenciam o documento, está o livro que acompanha o disco e que possui uma dedicatória do compositor a tinta: "A Mario de Andrade // atenciosamente / Hekel Tavares // Natal de 1934".

Mário de Andrade foi poeta, romancista, musicólogo, historiador críticodearteefotógrafo.Elefoium dospaisdomodernismobrasileirocoma publicação, em 1922, de um livro de poesia moderna Pauliceia desvairada. Ele foi também um dos organizadores da Semana de Arte Moderna de 1922, em São Paulo, que reformulou a literatura e as artes visuais no Brasil, e seu romance mais famoso é Macunaíma (1928).

O disco, e particularmente o livro, que possui ilustrações produzidas por Alceu de P. Penna, é rico em cores, imagens e aplicações, é um exemplar que necessita de cuidados especiais em virtude da hibridicidade de suportes e da delicadeza de impressão. Nesse sentido, com o objetivo de melhor caracterizar esses objetos, iniciou-se a análise dessa obra com a técnica de fluorescência de raios $X$ (XRF). Nesta análise, utiliza-se um sistema portátil que permite análises in situ, isto é no próprio laboratório do IEB. Neste trabalho determinou-se a composição dos pigmentos presentes nas ilustrações do livro, e também a composição do disco. Entre os pigmentos analisados, o dourado, por exemplo, possui como elemento chave, o Cobre $(\mathrm{Cu})$, utilizado para dar brilho à tinta. $\mathrm{O}$ disco tem uma composição complexa, com vários elementos como: Cálcio (Ca), Titânio (Ti), Ferro (Fe), Cobre (Cu), Zinco (Zn), Arsênio (As), Rubídio $(\mathrm{Rb})$, Estrôncio ( $\mathrm{Sr}$ ), Zircônio ( $\mathrm{Zr}$ ) e Chumbo (Pb). Sendo assim, a análise e caracterização dos elementos quimicos presentes nos materiais podem fornecer informações importantes para a sua documentação, além de ajudar na preservação e restauração da obra.

\section{O método XRF}

A espectroscopia de fluorescência de raios $\mathrm{X}$ baseia-se na emissão de raios $\mathrm{X}$ característicos dos elementos presentes na amostra, a partir de excitação por meio de um feixe de raios $X$ criado por um tubo de raios $X$. Os raios $\mathrm{X}$ emitidos da amostra são coletados por um detector específico de Si que gera um espectro de intensidade em função da energia.

A fluorescência de raios X (XRF) é uma técnica de análise elementar que vem sendo usada há mais de meio século para caracterizar os materiais de interesse cultural. Suas primeiras aplicações nesse campo datam 
de 1950, quando foi utilizada para analisar pequenos objetos metálicos ${ }^{7}$, vidros $^{8}$, jade $^{9}$ e pigmentos ${ }^{10}$ e 11. Desde o início essa técnica vem sendo usada de modo não-destrutivo, mas as potencialidades dessa metodologia aumentaram quando foram otimizados os detectores (fabricação de detectores semicondutores $)^{12}$ e 13 e quando foram desenvolvidos detectores semicondutores com resfriamento termoelétrico, que permitiu a construção de sistemas compactos, isto é, portáteis, para realizar análises in situ. Desse modo a técnica analítica XRF é não destrutiva, multielementar e simultânea, permitindo, assim, a determinação de elementos químicos com número atômico $\geq 12$ (Magnésio-Mg). Nessa técnica, podem-se identificar os raios $X$ característicos das transições eletrônicas nos orbitais K, L ou M dos elementos (Figura 1), que estão presentes em uma amostra particular ${ }^{14}$. Utilizando-se fontes de excitação, como tubos de raios $X$ ou fontes radioativas emissoras de raios $X$ ou raios gama, que forneçam energias adequadas para provocar a excitação dos elétrons das camadas internas do átomo, é possível induzir transições eletrônicas nos níveis mais internos dos átomos com a consequente emissão de raios $\mathrm{X}$ característicos (Figura 1). Com o emprego de detectores específicos, capazes de realizar a separação em energia dos diferentes raios $\mathrm{X}$ característicos, obtém-se um espectro de energia a partir do qual se realiza a identificação e quantificação dos elementos.

7 KRAAY, C. M., “The composition of electrum coinage”, Archaeometry, v.1, p. 21-23, 1958.

8 BANKS, M.; ELPHINSTONE, N.; HALL, E. T. "Bristol blue glass”, Archaeometry, v. 6, p. 26-zo, $196 z$.

9 HALL, E. T.; BANKS, M.S. J. M. Stern. "Uses of X-ray fluorescence analysis in archaeology", Archaeometry, v. 7, p. 84-89, 1964.

10 GETTENS R. J.; FITZHUGH, E. "Azurite and blue verditer", Studies in conservation, v. 11, p. 54-61, 1966.

1 GETTENS, R. J; KUHN, H.; CHASE, W. T. "Lead white”, Studies in conservation, v.12, p. $125^{-139,1967 . ~}$

12 CESAREO, R. et al, "Non destructive analysis of chemical elements in paintings and enamels", Archaeometry, v. 14, p. 65-78, $197^{2}$.

13 HALL, E. T.; SCHWEIZER F.; TOLLER, P. A. "X-ray fluorescence analysis of museum objects: a new instrument”, Archaeometry, v. 15, p. 53-78, 1973.

14 BERTIN, E. P. "Principles and practice of X-ray spectrometric Analysis", Plenum Press: Londres, 1975 . 

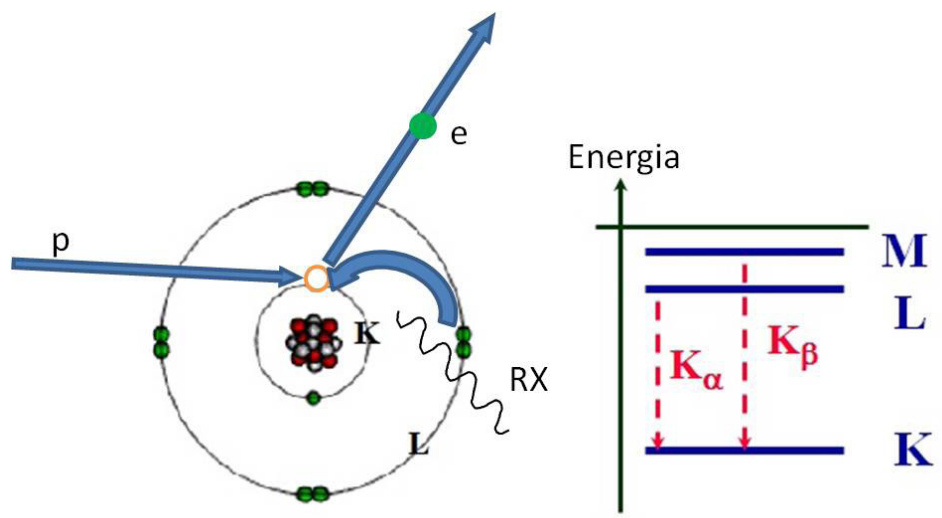

Figura 1. Desenho esquemático da interação de prótons com elétron do orbital K, sua remoção e o preenchimento da lacuna por um eletron do orbital L, e a emissão de Raio X em virtude dessa transição eletrônica do orbital L para K.

\section{Materiais e métodos}

O sistema portátil de XRF (PXRF) utilizado (Figura 2) é composto por um tubo de raios $\mathrm{X}$ com anodo de prata - operando a $28 \mathrm{kV}$ e 4,5 $\mathrm{A}$ com filtro de 50 micra de prata, um detector Si-PIN XR-100CR da Amptek e um sistema para posicionamento do detector e tubo de raios $\mathrm{X}$ com graus de liberdade de rotação e translação em relação ao objeto a ser medido ${ }^{15}$. Esse sistema foi aplicado na análise dos pigmentos presentes nas ilustrações do livro, no papel e também no disco de vinil.

A análise dos pigmentos é importante, pois permite identificar quais os pigmentos que foram utilizados pelo artista e como foram misturados para produzir determinado tom. Essa análise é igualmente importante nos processos de restauro, uma vez que ajuda a diferenciar os pigmentos originais dos pigmentos de restauro realizados posteriormente, auxiliando o profissional tanto na escolha dos pigmentos para retocar a pintura, quanto na remoção de camadas espúrias. Além disso, permite ainda auxiliar na conservação do objeto, já que alguns pigmentos podem ser sensíveis à luz, à umidade, a poluentes atmosféricos ou ao calor, e isso pode demandar ambientes especiais para armazenamento ou, ainda, cuidados na exposição de uma obra. Semelhantemente, a caracterização do papel e do disco de vinil é necessária para melhorar caracterização e para auxiliar no processo de conservação e restauro.

15 IKEOKA, R. A. et. al. "PXRF and multivariate statistics analysis of pre-colonial pottery from northeast of Brazil”, X-ray spectrometry, v. 41, p. 12-15, 2012. 


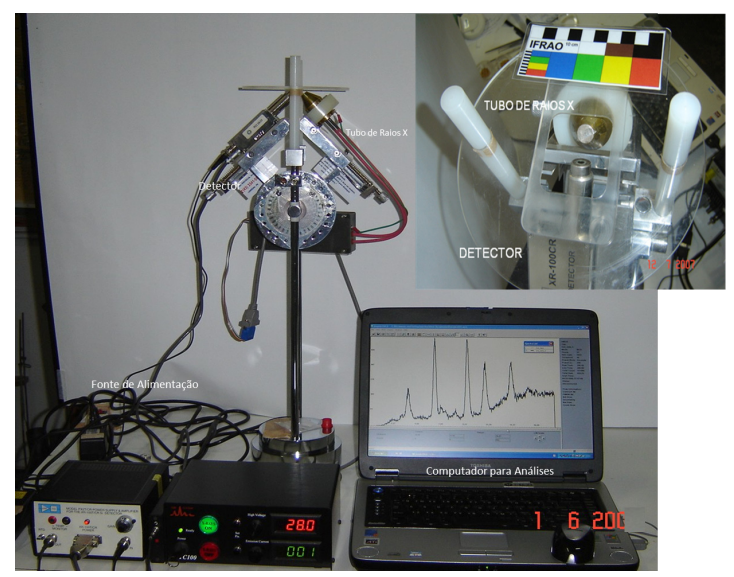

Figura 2. Fotografia do arranjo experimental de Fluorescência de raios $\mathrm{X}$ portátil da Universidade Estadual de Londrina.

Os discos eram prensados e processados usando-se os mais variados tipos de materiais, como substâncias alcatroadas, bases metálicas, de vidro, recobertas com vernizes e acetatos, misturas de resinas naturais tipo goma-laca e, por fim, algumas das primeiras resinas sintéticas fenólicas, como o "condensite". A partir de 1930, os engenheiros relacionados com a indústria fonográfica tentavam conseguir gravações com longo tempo de duração e sonoridade de alta fidelidade. Entretanto, esse intento foi conseguido somente em 1948, com o aparecimento do disco com microssulco, desenvolvido nos laboratórios da Columbia Broadcasting System (CBS), Estados Unidos, baseado na pesquisa pioneira do Dr. Peter Goldmark. Esse novo processo de gravação, cuja prensagem agora era feita em um novo tipo de material conhecido como "vinylite", trazia diversas vantagens quando comparado aos predecessores; entre as principais se destacam a reprodução com longa duração - daí o seu nome "Long playing" -, melhora na faixa dinâmica, resposta de frequência de alta fidelidade, operação com fonocaptores com baixíssima pressão de trilhagem, maior resistência ao desgaste e ausência de ruído superficial ${ }^{16}$.

A seguir, na Figura 3, temos três páginas do livro O sapo dourado, que foi analisado por XRF.

16 FAZANO, Carlos A. A origem da indústria fonografica. Disponível em: <http://fazano. pro.br/portiz6.html>. Acesso em 27 jul. 2011. 

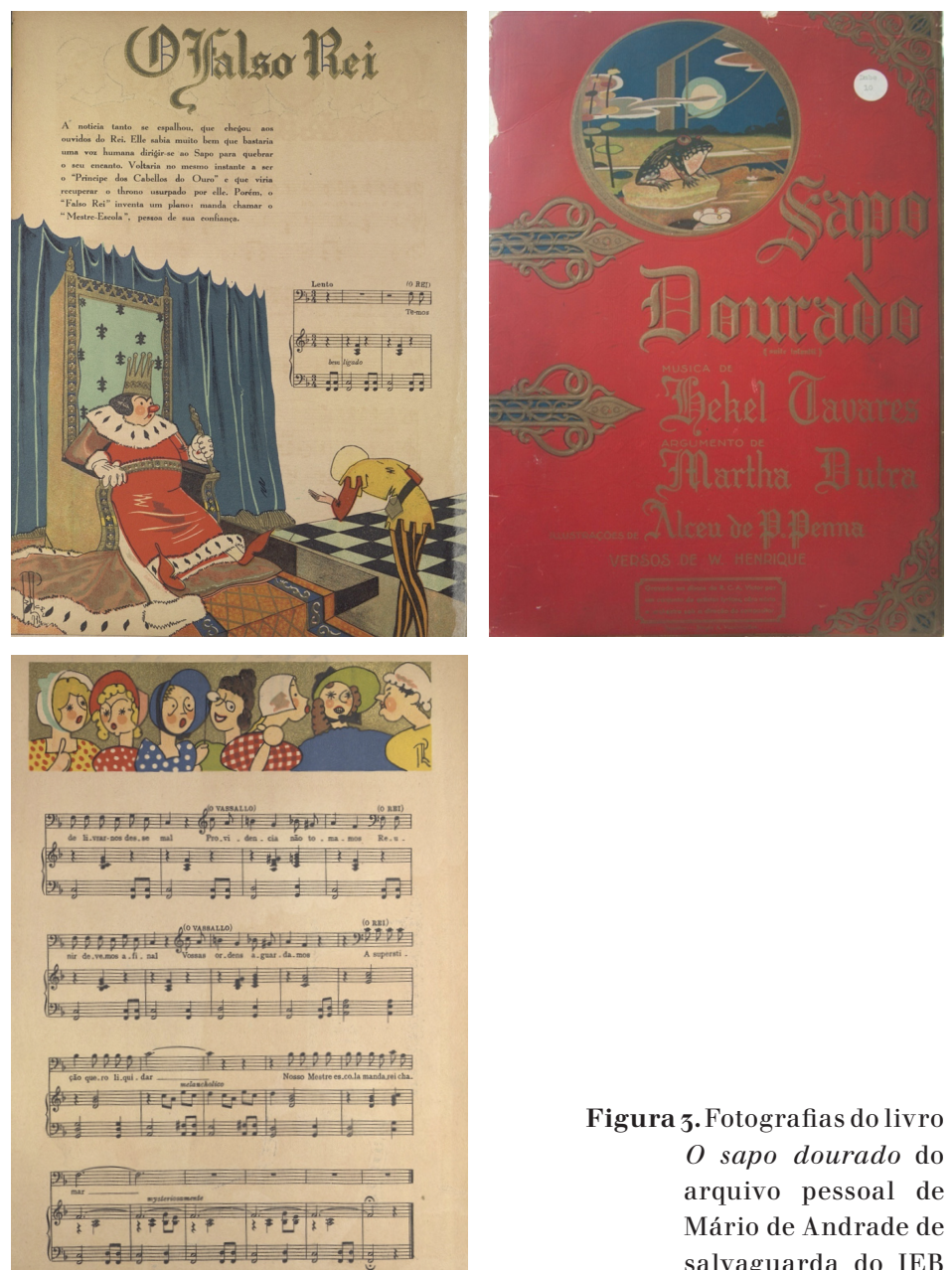

Figura z. Fotografias do livro O sapo dourado do arquivo pessoal de Mário de Andrade de salvaguarda do IEB

\section{Análise e resultados}

Foram analisados pela técnica XRF os seguintes pontos:

- Pigmento preto - Pontos 1 e 4

- Pigmento dourado - Ponto 2

- Pigmento vermelho - Ponto 5

- Pigmento azul - Ponto 6

- Papel-Pontos 3 e 9

- Disco - Ponto 8

- Fundo 
A Figura 4 exemplifica o uso do sistema portátil XRF para as medidas in situ no laboratório de Conservação e Restauro do IEB.
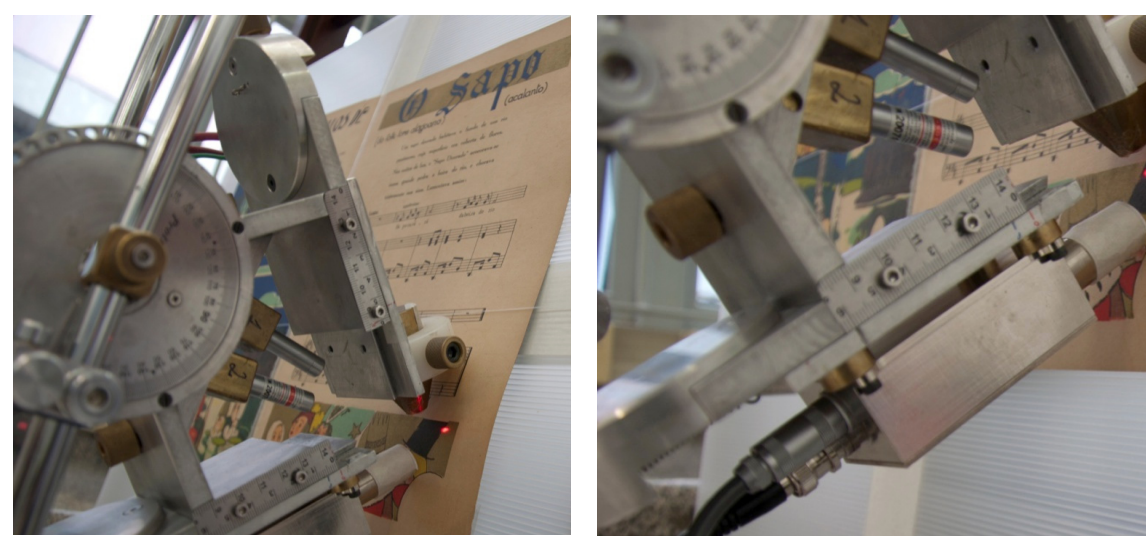

Figura 4. Pontos analisados com o sistema XRF portátil. Esquerda: Ponto 1 pigmento preto. Direita: Ponto 2 pigmento dourado

O sistema possui um conjunto de lasers para melhor posicionamento do objeto a ser estudado. O tempo de cada medida foi de 500 segundos, e dois espectros típicos da medida XRF são apresentados na Figura 5. Basicamente, os elementos presentes nos pigmentos são Cromo (Cr), Ferro (Fe), Cobre (Cu), Zinco (Zn) e Chumbo (Pb). Como se pode observar na Figura 5, o pigmento dourado possui uma grande quantidade de cobre e zinco em relação ao pigmento preto, e o mesmo ocorre para o pigmento vermelho. O pigmento preto possui muito mais chumbo. Sendo assim, o pigmento preto poderia ser à base de Plattnerita $\left(\mathrm{PbO}_{2}\right), \mathrm{e}$ o pigmento dourado à base de pó da liga de cobre e zinco.
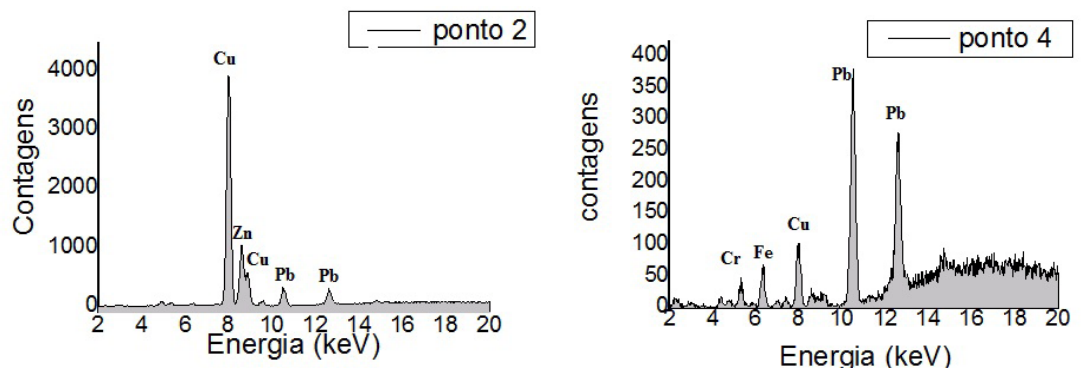

Figura 5. Espectros XRF típicos. Esquerda: Ponto 2 pigmento dourado. Direita: Ponto 4 pigmento preto 
O pigmento branco de chumbo (2PbCO3. $\mathrm{Pb}(\mathrm{OH}) 2)$ foi desenvolvido pelos gregos e continuou a ser utilizado até cerca de 1960. Esse pigmento é encontrado nas colorações brancas ou em mistura de alguns pigmentos para dar tonalidades diferentes e foi muito utilizado pelos artistas até o século XIX, quando surgiu o branco de zinco ${ }^{17}$.

A análise do disco também apresentou o elemento ferro em grande quantidade além dos elementos cobre, zinco e chumbo (em menor quantidade), mas evidenciou outros elementos como cádmio, titânio, arsênio, rubídio, estrôncio e zircônio, que estão relacionados ao processo de manufatura do disco de vinil. Depois do ferro, o arsênio é o elemento que apresenta maior contagem. O espectro típico, bem como a foto com a medida sendo realizada no disco de vinil, são apresentados na Figura 6.
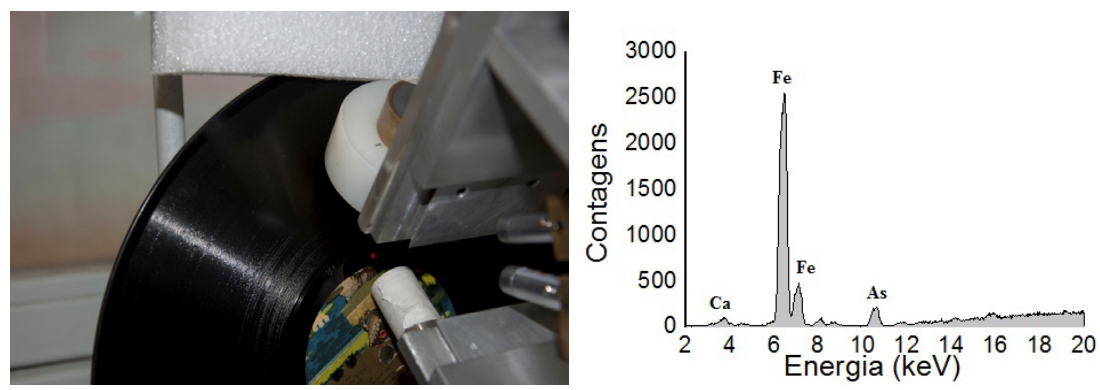

Figura 6. Esquerda: análise do disco com o sistema XRF. Direita: Espectro XRF do ponto do disco analisado.

Esses elementos identificados no disco são consistentes com os materiais utilizados no processo de manufatura anterior a 1948, reforçando a idade cronológica da fabricação do disco. A análise do papel permitiu identificar os elementos ferro, níquel e cobre. Em um dos pontos de papel analisado (ponto 3), há uma maior quantidade de ferro em relação ao outro ponto de papel analisado (ponto 9). Provavelmente, a quantidade maior desse elemento está relacionada à coloração mais escura no ponto 3, provavelmente por conta da oxidação do papel neste ponto.

Os elementos identificados, bem como a área líquida (descontada do fundo) determinada em cada pico dos respectivos espectros, são listados de maneira resumida na Tabela 1 . O fundo listado na tabela se refere ao suporte utilizado para posicionar as folhas de papel do livro na frente do sistema de PXRF e apresenta apenas o níquel, em virtude da interação

17 SILVA, W. D. da; APPOLONI, C. R. "Pigmentos: propriedades físicas, químicas e o período histórico de utilização”, LFNATEC (Publicação Técnica do Laboratório de Física Nuclear Aplicada, ISSN 2178-4507), v. 13, n. ol, 2009, 12 p. Disponível em: <http://www.fisica.uel.br/gfna/publictec.html>. Acesso em 1 set. 2012. 
dos raios $\mathrm{X}$ espalhados com o níquel do tratamento superficial do sistema de suporte para o detector e o tubo de raios X. Da mesma maneira, o níquel detectado nos outros pontos tem esta origem e não está relacionado a pigmentos, exceto no caso do disco que não teve esse suporte.

Tabela 1: Elementos e áreas dos picos dos diferentes pontos analisados

\begin{tabular}{|c|c|c|c|c|c|}
\hline Elemento & Ponto 1 & Ponto 2 & Ponto 3 & Ponto 4 & Ponto 5 \\
\hline Cálcio & Preto & Dourado & Papel & Preto & Vermelho \\
\hline Titânio & 43 & 35 & - & 135 & 77 \\
\hline Vanádio & 235 & 756 & - & 70 & 89 \\
\hline Cromo & 158 & 366 & - & 345 & 267 \\
\hline Ferro & 382 & 359 & 432 & 597 & 557 \\
\hline Níquel & 236 & 271 & 116 & 125 & 150 \\
\hline Cobre & 3616 & 40953 & 197 & 1031 & 19572 \\
\hline Zinco & 817 & 9554 & 46 & 219 & 4495 \\
\hline Arsênio & - & - & - & - & - \\
\hline Rubídio & - & - & - & - & - \\
\hline Estrôncio & - & - & - & - & - \\
\hline Zircônio & - & - & - & - & - \\
\hline Chumbo & 1598 & 3404 & - & 3993 & 2533 \\
\hline
\end{tabular}


Tabela 1 (continuação): Elementos e áreas dos picos dos diferentes pontos analisados

\begin{tabular}{|c|c|c|c|c|}
\hline Elemento & Ponto 6 & Ponto 8 & Ponto 9 & Fundo \\
\hline Cálcio & Azul & Disco & Papel & \\
\hline Titânio & - & 1002 & - & \\
\hline Vanádio & 88 & 181 & - & \\
\hline Cromo & 0 & - & - & \\
\hline Ferro & 110 & 40030 & 74 & \\
\hline Níquel & 104 & 68 & 173 & \\
\hline Cobre & 255 & 966 & 306 & \\
\hline Zinco & 73 & 435 & 128 & \\
\hline Arsênio & - & 2700 & - & \\
\hline Rubídio & - & 489 & - & \\
\hline Estrôncio & - & 638 & - & \\
\hline Zircônio & - & 590 & - & \\
\hline Chumbo & 791 & 533 & - & \\
\hline
\end{tabular}

\section{Conclusões}

As medidas realizadas permitiram obter indicações sobre a composição dos pigmentos utilizados na manufatura do livro e do disco. Tais pigmentos são consistentes com aqueles empregados no início do século XX, assim como a composição do disco é compatível com aquela reportada na literatura para o material empregado antes de 1948. Esses resultados são importantes para procedimentos de conservação e restauro desses materiais.

\section{Agradecimentos}

Os autores gostariam de agradecer a Mira Serrer Rufo, Indrani Taccari, Lúcia Helena de Souza, Maria Izilda Fonseca Leitão, Mônica A. G. Silva Bento, Elisabeth Marin Ribas. Ao CNPq e FAPESP. Este trabalho foi um desdobramento do projeto FAPESP ${ }^{0}$ 2009/54785-3, coordenado pela Profa. Dra. Flávia Camargo Toni. 